\title{
Explaining the rise in antidepressant prescribing: a descriptive study using the general practice research database
}

\author{
Michael Moore, senior lecturer, ${ }^{1}$ Ho Ming Yuen, medical statistician, ${ }^{2}$ Nick Dunn, director of GP teaching, ${ }^{3}$ Mark \\ A Mullee, director, research design service south central, ${ }^{2}$ Joe Maskell, data manager, ${ }^{2}$ Tony Kendrick, \\ professor of primary medical care ${ }^{1}$
}

University of Southampton, Aldermoor Health Centre, Southampton S016 5ST

${ }^{2}$ Public Health Sciences and Medical Statistics, University of Southampton, Southampton S016 6YD

${ }^{3}$ Division of Medical Education, School of Medicine, University of Southampton, Southampton S016 7PX

Correspondence to: Michael Moore mvm198@soton.ac.uk

Cite this as: BMJ 2009;339:b3999 doi:10.1136/bmj.b3999

\section{ABSTRACT}

Objective To explore the reasons behind the recent increase in antidepressant prescribing in the United Kingdom.

Design Detailed retrospective analysis of data on general practitioner consultations and antidepressant prescribing.

Data source Data were obtained from the general practice research database, which contains linked anonymised records of over 3 million patients registered in the UK. Data were extracted for all new incident cases of depression between 1993 and 2005.

Review methods Detailed analysis of general practitioner consultations and antidepressant prescribing was restricted to 170 practices that were contributing data for the full duration of the study.

Results In total, 189851 people within the general practice research database experienced their first episode of depression between 1993 and 2005, of whom 150825 (79.4\%) received a prescription for antidepressants in the first year of diagnosis. This proportion remained stable across all the years examined. The incidence of new cases of depression rose in young women but fell slightly in other groups such that overall incidence increased then declined slightly (men: 7.83 cases per 1000 patient years in 1993 to 5.97 in 2005, women: 15.83 cases per 1000 patient years in 1993 to 10.06 in 2005). Antidepressant prescribing nearly doubled during the study period-the average number of prescriptions issued per patient increased from 2.8 in 1993 to 5.6 in 2004. The majority of antidepressant prescriptions were given as long term treatment or as intermittent treatment to patients with multiple episodes of depression.

Conclusions The rise in antidepressant prescribing is mainly explained by small changes in the proportion of patients receiving long term treatment. Previous clinical guidelines have focused on antidepressant initiation and appropriate targeting of antidepressants. To address the costly rise in antidepressant prescribing, future research and guidance needs to concentrate on appropriate long term prescribing for depression and regular review of medication.

\section{BACKGROUND}

Substantial increases in antidepressant prescribing have been observed in the United Kingdom over the past two decades. Data from the Prescription Pricing Authority show that antidepressant prescribing in England increased by $36 \%$ to 7.3 million items per quarter between 2000 and 2005, and costs increased by $20 \%$ to $£ 91$ million ( $€ 100$ million; $\$ 145$ million) per quarter. ${ }^{1}$ Prescribing of selective serotonin reuptake inhibitors increased by $45 \%$ during this period and accounted for half of all prescriptions and costs for antidepressant drugs. Further rises, predominantly in the prescription of selective serotonin reuptake inhibitors, have occurred since these data were released, although costs have fallen since 2005 because several key patents have expired. ${ }^{2}$ In addition, evidence exists for rises in antidepressant prescribing since the middle of the $1970 \mathrm{s.}^{3}$ These trends are not limited to the UK: similar rises have been described in other Western nations such as Australia and Canada. ${ }^{4-9}$

Various explanations have been proposed to decipher these long term trends, including improved adherence to guidelines-resulting in longer initial courses of antidepressants - and a lower threshold for prescribing. A study combining longitudinal data from prescribing surveys and data from general practices in Scotland explored four potential explanations: increased incidence; increased prevalence; increased care seeking behaviour by patients; and improved identification of depression by general practitioners. ${ }^{10}$ Despite confirming the increase in antidepressant prescribing, the authors were unable to find any changes in incidence or prevalence of depression, or any change in help seeking behaviour. Nor did general practitioners record a higher number of diagnoses of depression.

Another plausible explanation is that long term repeat prescribing of antidepressants has increased in recent years. A cross sectional survey of general 
practice records from 2002 and 2004 highlights the relative importance of chronic prescribing. ${ }^{11}$ In this study, depression and mixed anxiety and depression accounted for $61 \%$ of all prescriptions for antidepressants. New prescriptions for antidepressant medication initiated in primary care have very high discontinuation rates, ${ }^{12} 13$ and, therefore, mainly comprise short courses. Nevertheless, depression and mixed anxiety were associated with long term use of antidepressants in this study, with an average duration of five to seven years. The implication is that long term prescriptions have a greater influence on average treatment length than incident prescribing.

The general practice research database (GPRD) is an internationally recognised resource that contains linked anonymised primary care records of over 3 million patients registered in the UK. The database is widely used for pharmacoepidemiological research and allows follow-up of large cohorts of users of specific drugs. ${ }^{14}$

The objective of this study was to investigate using the GPRD changes in the patterns of antidepressant prescribing over time. We also aimed to investigate whether the widely reported increase in antidepressant prescriptions in recent years is the result of any of the following reasons: an increase in the incidence of new cases of depression (which may reflect improved recognition or lower thresholds for diagnosis); an increase in the proportion of new cases of depression for whom antidepressants are prescribed; an increase in the duration of prescribing for new cases of depression (in line with guidelines recommending treatment for four to six months after remission ${ }^{15}$ ); or an increase in long term prescribing of antidepressants overall (either repeated courses to treat recurrences or continuous prescribing to prevent recurrence).

\section{METHODS}

We identified in the GPRD incident cases of new depression that arose between 1993 and 2005. Patients were included if they received a first ever antidepressant prescription for depression diagnosed up to 180 days before or 90 days after the prescribing event, or received a first ever diagnosis of depression without an associated prescription for antidepressants.

New practices joined the GPRD during data collection, so the denominator-that is, the number of patients registered in the GPRD-varied from 1.5 million in 1993 to a maximum of 3 million in 2001, with an approximate total of 29 million registered patients over the 13 year study. In order to better understand the changes in the data, we restricted our detailed analysis to practices that remained in the database throughout the period of study and submitted data that met the GPRD quality criteria. Although patients can leave individual practices, using only the data from these practices will provide more stable interpretation of trends.

Patients entered into a cohort on the basis of the year of their first ever diagnosis of depression and then remained in the cohort for the remainder of the study period. They may never have received a prescription for antidepressants, had a single episode of treated depression, had multiple episodes of depression, or received continuous treatment for depression. The cohort of patients being followed in 1993 comprised the incident cases of first ever episode depression in that year only, whereas the cohort in following years included patients from previous years as well as the incident cases that year. Once patients had entered the cohort they then remained in the cohort until the end of the study period or until they were transferred out for other reasons (for example, death), while new incident cases were continually added. Thus in later years the total cohort included a mixture of incident cases, prevalent cases, and new episodes occurring in those who had entered the cohort previously.

We used patient years at risk as the denominator to calculate the overall incidence of first episode of depression. For example, someone who was registered in the GPRD for only six months of the year would contribute 0.5 years to the denominator. Prescribing events were episodes of antidepressant prescribing, regardless of prescription duration. Duration of prescription was calculated using an algorithm described in the detailed methods online.

To address our study questions and examine more closely trends occurring within the cohort, we divided patients into five pre-defined treatment pattern groups on the basis of treatment in the five years following first diagnosis (five year follow-up was possible only for those diagnosed between 1993 and 2001). The five pre-defined treatment pattern groups were:

- Chronic treatment: patients who received a prescription in the year of first diagnosis and in every year after that, for five years

- Intermittent treatment: patients who received a prescription in the year of first diagnosis and at least one subsequent year

- Short term treatment: patients who received a prescription in the year of first diagnosis but not in any subsequent years

- Delayed treatment: patients who received no prescription in the year of first diagnosis but did receive a prescription in any subsequent year up to five years of follow-up

- No treatment: patients with depression who never received a prescription within five years of follow-up.

These five treatment pattern groups are mutually exclusive - that is, each incident case could only fall into one of the five groups followed up for a maximum of five years from the year of their first diagnosis. Patient data were included only when information for all five years was available. The data analyses for this research were undertaken using version 9.1 of the SAS System for Windows (SAS Institute Inc; Cary, NC, USA).

\section{RESULTS}

\section{Diagnostic events}

The stable dataset included 170 practices and 1.7 million registered patients. Patients in the stable cohort did 


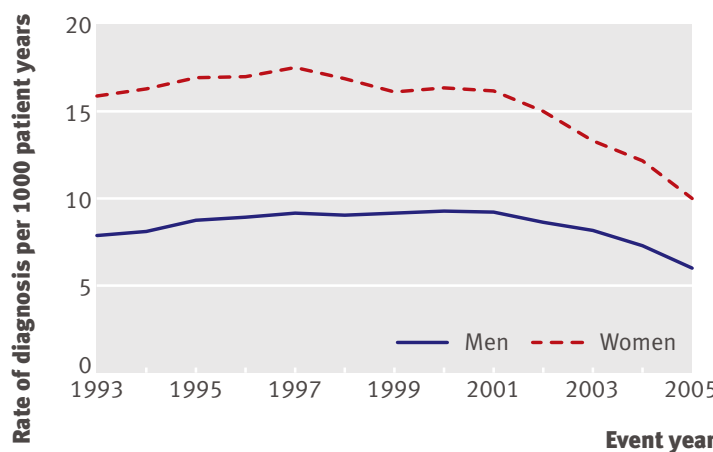

Fig 1 | Incidence of first event of depression per 1000 patient years in the 170 stable practices

not differ from the whole cohort in their age and sex distribution. Within the stable cohort, 632044 diagnoses of depression were recorded. The top 20 most frequently used diagnostic labels are listed in web table A.

In total, 189851 patients had their first diagnosis of depression between 1993 and 2005. The top 20 most common diagnostic labels according to patients' first ever diagnosis are listed in web table B. No major differences were apparent between the whole cohort and the stable practices in use of diagnostic labels; for instance, the top 20 labels accounted for $97.3 \%$ of all diagnoses in the whole cohort compared with $96.9 \%$ in the stable practices.

\section{Incidence of depression}

Figure 1 illustrates the incidence of first episodes of depression per 1000 patient years in the 170 stable practices. In 1993, the incidence of new cases depression was around twice as high in women as in men $(9493 / 599849(15.8 \%)$ v 4573/584 049 (7.8\%)). Overall incidence across each gender declined slightly between 2001 and 2005 (men: 7.83 cases per 1000 patient years in 1993 to 5.97 in 2005 , women: 15.83 cases per 1000 patient years in 1993 to 10.06 in 2005 ; web table C).

Incidence rates were slightly higher in the whole cohort than in the stable practices. For example, in 1993, the incidence in men was $9.3 \%$ for the whole cohort compared with $7.8 \%$ in stable practices (6373/ 683586 and 4573/584049, respectively), and in females these values were $18.3 \%$ and $15.8 \%$, respectively (12 868/709 905 and 9493/599 849).

The data were then further divided into four age bands to demonstrate the relation between age and incidence of first episode of depression (figs 2 and 3; web table $\mathrm{C}$ ). These data show that the highest incidence of the first episode of depression was in women aged between 18 years and 30 years. Over the 13 year period, the incidence in women in this age group rose from 23.8 per 1000 patient years in 1993 to peak at 37.7 per 1000 patient years in 2001, before falling again to 26.4 per 1000 patient years in 2005. Thus, the incidence of new cases of depression rose in young women but fell slightly in other groups such that overall incidence declined slightly.

Although the incidence in men was always lower than that in women, the change over time in each age group was similar. The highest incidence of first depression was seen in the 18-30 years age group for both men and women.

\section{Prescribing events}

The prescription data for the stable cohort consisted of 2108311 prescribing events among 153931 patients. We combined these data with the diagnosis data to look at prescriptions specifically in patients who had a diagnosis of depression. According to these combined data, there were 2093737 prescribing events in 189851 patients who were diagnosed as depressed. Of those patients diagnosed as depressed, 153914 (81.1\%) received at least one prescription for antidepressants.

The proportion of patients with depression who received a prescription for an antidepressant in the year of their first diagnosis was then examined (web table D). The majority $(150825(79.4 \%))$ received a prescription in the year of their first diagnosis. The proportion who received a prescription in the year of diagnosis was relatively constant at around $80 \%$, then fell slightly after 2002 to $74 \%$ in 2005 . The proportion of patients who received ongoing prescriptions, however, increased over the course of the study (web table D). The proportion of patients who remained on treatment in the second year after diagnosis, for example, increased from $40.6 \%$ in 1994 to peak at $51.4 \%$ in 2004 (4533/1159 and 6430/12502, respectively). This trend was also observed within in the third, fourth, and fifth year after diagnosis, although the magnitudes were relatively smaller.

The average number of prescriptions issued per patient in each year of the study period was then determined. The number of prescriptions rose from 2.8 per patient in 1993 to 5.6 per patient in 2004 . However, in later years new patients were continually added so that the cohort included a mixture of incident cases, prevalent cases, and new episodes occurring in those who had entered the cohort previously. Therefore, the increase in the number of prescriptions is likely to have arisen from a change in the nature of the cohort

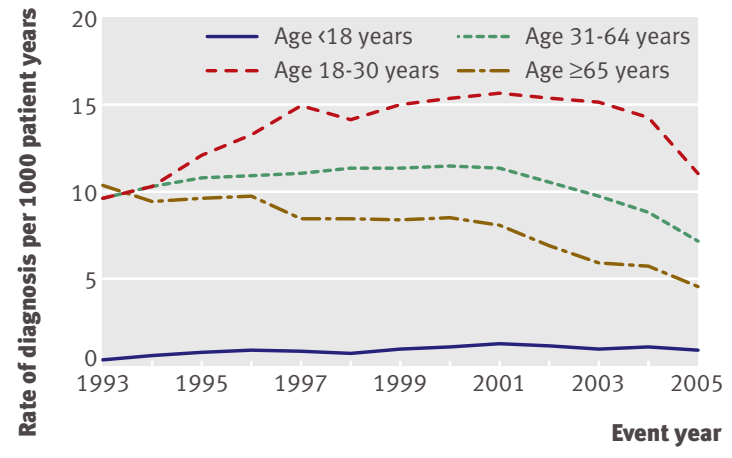

Fig 2 Rate of diagnosis of first episode of depression per 1000 patient years by age in male patients 


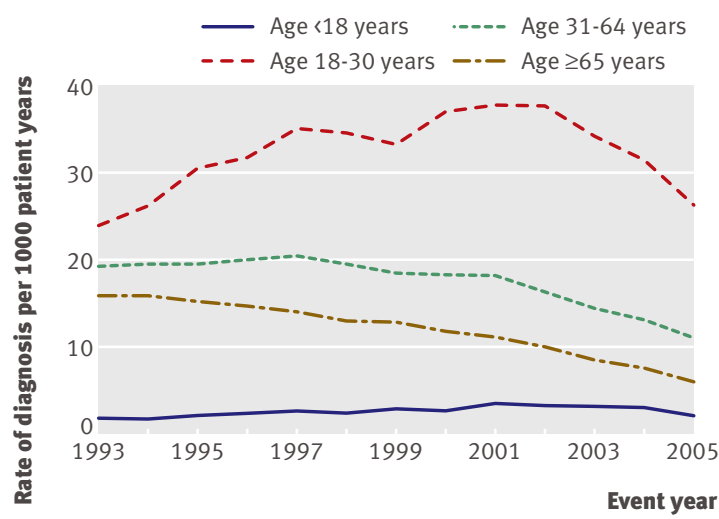

Fig 3 Rate of diagnosis of first episode of depression per 1000 patient years by age in female patients

to include a mixture of incident and prevalent cases. We were able to confirm that the observed increase in prescription episodes arose because those who had been followed for a longer period after the first episode of depression had an increasing number of prescription episodes over time.

\section{Prescription duration}

The data for prescription duration in the first year of diagnosis show a small decrease in short term prescriptions (<30 days) from $87.1 \%$ of all prescriptions in 1993 to $84.6 \%$ (in 2004(25909/29 737 and $25749 / 30444$, respectively). The proportion of short term prescriptions diminished with time from first year of diagnosis-for instance, to $77.8 \%$ after 5 years. Conversely, the proportion of prescriptions for 31-60 days rose from $12.3 \%$ in the year of diagnosis to $20.1 \%$ after 5 years.

Although the majority of prescriptions were for less than 30 days (fig 4), the proportion of patients who received such prescriptions decreased from $87.1 \%$ in 1993 to $78.2 \%$ in 2005 (25909/29 737 and 155360/ 198799 , respectively). The reverse trend was seen in the proportion of patients on a long duration prescription, which increased slightly over the period of study. The proportion of patients receiving a prescription for 31-60 days, for example, increased from 12.3\% in 1993 to $19.9 \%$ in 2005 (3668/29 737 and $39539 / 198779$, respectively), and the proportion on antidepressants for $61-180$ days went from $0.5 \%$ to $1.8 \%$ (151/29 737 and 3622/198 779). Again, this change is likely to arise from a change in the nature of the cohort to include a mixture of incident and prevalent cases.

\section{Patterns of treatment received}

Figure 5 shows the proportion of patients in each of the five treatment pattern groups-chronic treatment, intermittent treatment, short term treatment, delayed treatment, and no treatment. Between 1993 and 2001 there was a small but steady increase in the proportion of patients receiving intermittent treatment $(34.8 \%$ $(4894 / 14064) v 38.2 \%(5969 / 15645))$, together with a small rise in those receiving chronic treatment $(8.4 \%$ $(1181 / 14064) v 10.2 \%(1597 / 15645))$. The proportion of patients receiving short term treatment showed a corresponding decrease $(36.2 \% \quad(5084 / 14064) \quad v$ $31.6 \%(4936 / 15645))$. The proportion receiving delayed treatment or no treatment remained constant at around $1 \%$ and $19 \%$, respectively.

The average duration of individual prescriptions over five years was roughly similar for each treatment pattern group. As would be anticipated, the chronic treatment group generally had slightly longer average prescription duration (by 1-2 days).

Figure 6 shows that the chronic treatment group received a higher number of prescriptions than any other group - on average 35 prescriptions per patient over five years - and, therefore, received the bulk of the prescriptions numerically. There was also an increase in the average number of prescriptions per patient in the chronic treatment group: from 34 over five years to 36 over five years in the period studied which equates to approximately 60 days more treatment over the five year period in 2001-5 compared to 1993-7. Bearing in mind the increase in the proportion of patients in this group (from $8.4 \%$ to $10.2 \%$ ), the increase in the duration of prescriptions in the chronic treatment group is responsible for a substantial increase in the overall prescribing volume. A similar pattern was seen in the other treatment groups, but with a smaller impact on overall volumes.

\section{Total prescription days}

Table 1 shows the total number of prescription days over the five years from first diagnosis for each of the treatment pattern groups. The data show an overall increase in prescription duration from just under 2.9 million days in the patients studied between 1993 and 1997 to more than 4.3 million days in the those studied between 2001 and 2005. Chronic and intermittent prescribing together accounted for approximately $90 \%$ of the total prescriptions (for example, 270 005/288 249 in 1993). Although the number of prescription days increased over time for all treatment groups, small changes in the constitution of the chronic treatment and intermittent treatment groups were responsible

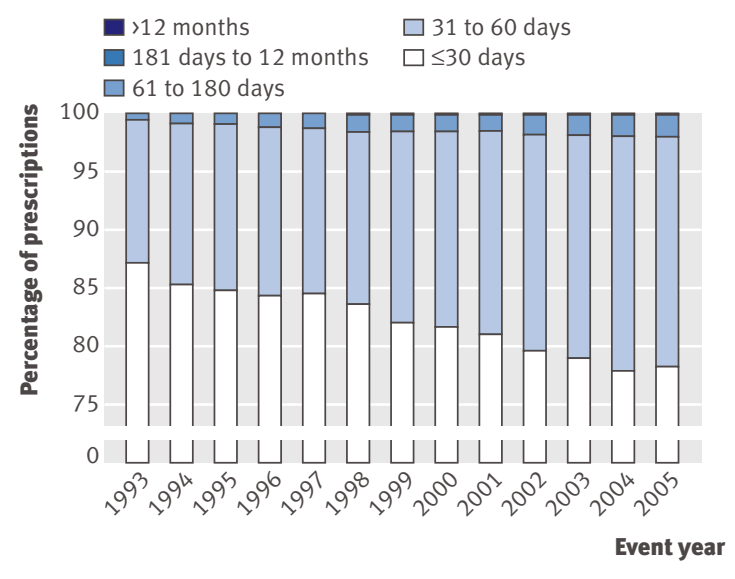

Fig $4 \mid$ Changes in the proportions of patients receiving prescriptions for a particular duration 


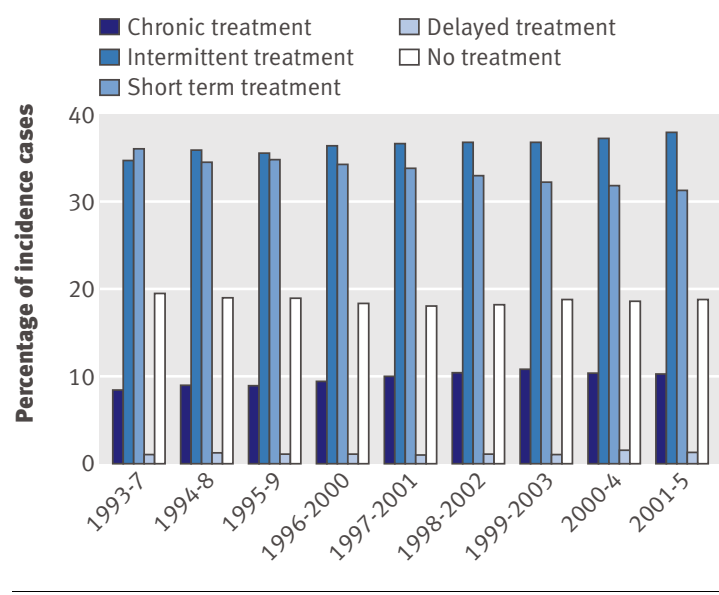

Fig $5 \mid$ Proportion of incident cases in each of the treatment pattern groups

for most of the increase in antidepressant prescribing over the duration of the study.

Total prescription days for each of the five treatment pattern groups is summarised in figure 7 . The volume of prescriptions attributed to long term prescribing (that is, the proportion of prescriptions in the chronic and intermittent groups) contributed the majority of the change in overall prescribing volume.

\section{DISCUSSION}

We have demonstrated that between 1993 and 2005, there was overall a small change in the incidence of new diagnoses of depression and a small increase in the mean duration of the antidepressant prescription for the first episode of depression. However, there were more marked trends within age and sex, including a large rise in the incidence of new diagnoses among young women and, to a lesser extent, among young men.

The majority of antidepressant prescriptions were given as long term treatment or to patients with multiple episodes of depression. Small increases both in the proportion of patients in these groups and in the duration of prescriptions made to such individuals accounted for a near doubling of the total volume of antidepressant prescribing between 1993 and 2005 . Although the proportion of new cases initially treated with antidepressants did rise slightly over this period, changes in recognition, case definition, or duration of initial prescription are unlikely to account for the dramatic increase in antidepressant prescribing. A key question remains: if the changes in antidepressant prescribing are accounted for by changes in the proportions of those in receipt of long term prescriptions, does this represent appropriate prescribing for those with chronic and relapsing disease according to current guidance or does it arise from a failure to discontinue antidepressants in those with milder illness, or both?

The majority of new episodes of depression were associated with antidepressant prescribing in the year of diagnosis $(79 \%)$, similar to other studies. ${ }^{91617}$ This proportion remained remarkably stable over the period studied, falling only in the last two years.

\section{Strengths and limitations of study}

The data on both diagnosis and prescribing in the GPRD are very reliable given the stability of the lists of registered patients and the healthcare system. The GPRD has no formal control on diagnostic categories though, which are determined by individual general practitioners. We did include all possible depression diagnostic codes in our study, but cannot exclude the possibility that some prescribing took place without any appropriate depression code. Also, prescribing records reflect the issue of prescriptions and not the dispensing or taking of the medication. Although the majority of prescribing takes place in primary care in the UK, medication may also be prescribed in other settings - for example, to hospital outpatients-but this is likely to contribute little overall in the UK.

In this study we were principally interested in patterns of prescribing rather than absolute numbers. Although the data are observational and we were unable to control for patient level confounders, we believe that this large dataset does give valuable insights into prescribing patterns in the UK. We can be less confident regarding the generalisability of our findings to other healthcare systems where antidepressant prescribing may not be so dominated by primary care physicians.

We restricted the analysis to practices that contributed data to the GPRD throughout the whole study period. We thought that although patients can leave individual practices, the stability provided by using the 170 stable practices would allow greater confidence in interpretation of trends within the data. Including all practices - some of which joined or left the database over the study period - would make trends in prescribing more difficult to interpret. The question arises as to whether the stable practices are representative of the whole practice population. Various factors reassure us that this is the case. Firstly, being a stable practice

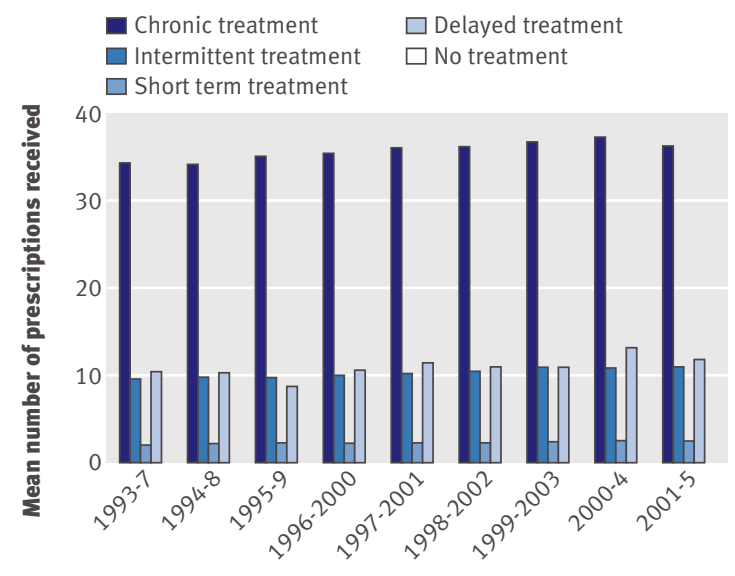

Fig 6 | Mean number of prescriptions received over the five year period following diagnosis for each of the five treatment pattern groups 


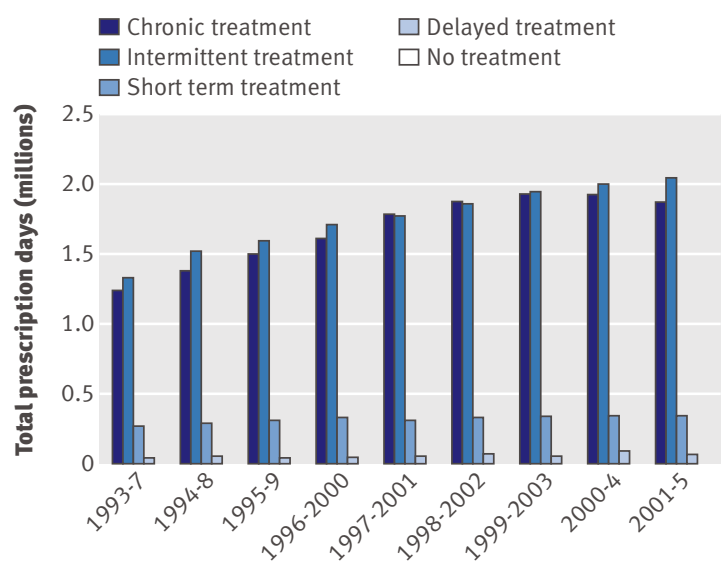

Fig $7 \mid$ Total number of antidepressant prescription days over five years from first diagnosis for the five treatment pattern groups

has no implications regarding the nature of the practice itself; the label just indicates that the practice was consistently contributing data that were regarded as up to standard for the entire study period. Secondly, there was no age or sex difference between the patients in the stable practices and those in the whole cohort, nor any major difference in diagnostic codes. We did identify that the incidence of depression appeared higher in the whole cohort. This disparity possibly arose as a result of incomplete data from some practices such that some cases of prevalent depression were included because of the absence of previous history codes.

Data that were entered into the GPRD system by general practitioners in the participating clinics were transferred to the GPRD organisation in batches. However, the decision on when to proceed with these transfers was made entirely by the clinics themselves. Hence, data collected close to the date on which we extracted data for this study, February 2006, might not contain the entire set from each clinic. This anomaly should only affect data from the final months of 2005 and is less likely to be a problem in the stable practices. We elected to include these data because a large number of observations were still available, and the incompleteness of the data should only have minimal effects on rates or proportions.

\section{Comparison with other studies}

Our data suggest that a small increase each year in the numbers of patients receiving repeat prescriptions accounts for a large increase in overall antidepressant prescribing volumes. Some support for these findings is found elsewhere in the literature.

In a Canadian study, the prevalence of new antidepressant prescriptions (defined in terms of no prescribing in the previous two years) rose between 1998 and 1999 then fell in 2004, whereas prescribing for prevalent cases doubled over the eight year study. ${ }^{16}$ These results are in accord with our findings and suggest that changes in the duration of prescribing are more important in determining overall prescription numbers than changes in the number of cases treated.

A US study similarly reported increased new prescribing of antidepressants to outpatients between 1987 and 1997, although no data were available on long term prescriptions. ${ }^{9}$ In another study, a cross section of general practitioner case notes was examined for patients on antidepressant prescriptions. The average duration of prescription was five to seven years where the prescribing indication was for mental health problems, suggesting that longer term prescribing dominates. ${ }^{911}$ Other previous studies have concentrated on the initial prescribing decision. ${ }^{17-19}$

National policy in the UK has focused on targeting antidepressants to patients with more severe symptoms by incentivising the use of questionnaire measures of severity before treatment through the UK general practice quality and outcomes framework..$^{20}$

A recent study of prescribing patterns following initiation of the framework showed appropriate targeting of antidepressants. ${ }^{21}$ The proportion of patients with a new diagnosis prescribed an antidepressant $(79 \%)$ was very similar to that in our study. This raises the question of how much influence the use of questionnaires actually has on the prescribing decision. It has already been established that patients are anxious regarding discontinuation of antidepressants and

\begin{tabular}{|c|c|c|c|c|c|c|c|c|c|c|}
\hline \multirow[b]{3}{*}{$\begin{array}{c}\text { Treatment } \\
\text { pattern group }\end{array}$} & \multirow[b]{3}{*}{$\begin{array}{c}\text { Total number } \\
\text { of patients }\end{array}$} & \multicolumn{9}{|c|}{ Five year follow-up from year of first diagnosis } \\
\hline & & 1993-1997 & $\begin{array}{l}1994- \\
1998\end{array}$ & $\begin{array}{l}1995- \\
1999\end{array}$ & $\begin{array}{l}1996- \\
2000\end{array}$ & $\begin{array}{l}1997- \\
2001\end{array}$ & $\begin{array}{l}1998- \\
2002\end{array}$ & $\begin{array}{l}1999- \\
2003\end{array}$ & $\begin{array}{l}2000- \\
2004\end{array}$ & 2001-2005 \\
\hline & & $\begin{array}{l}\text { Number of } \\
\text { prescription } \\
\text { days }\end{array}$ & $\begin{array}{l}\text { Number of } \\
\text { prescription } \\
\text { days }\end{array}$ & $\begin{array}{l}\text { Number of } \\
\text { prescription } \\
\text { days }\end{array}$ & $\begin{array}{l}\text { Number of } \\
\text { prescription } \\
\text { days }\end{array}$ & $\begin{array}{l}\text { Number of } \\
\text { prescription } \\
\text { days }\end{array}$ & $\begin{array}{l}\text { Number of } \\
\text { prescription } \\
\text { days }\end{array}$ & $\begin{array}{l}\text { Number of } \\
\text { prescription } \\
\text { days }\end{array}$ & $\begin{array}{l}\text { Number of } \\
\text { prescription } \\
\text { days }\end{array}$ & $\begin{array}{l}\text { Number of } \\
\text { prescription } \\
\text { days }\end{array}$ \\
\hline Chronic & 13783 & $\begin{array}{c}1239600 \\
(43.0 \%)\end{array}$ & $\begin{array}{c}1379889 \\
(42.6 \%)\end{array}$ & $\begin{array}{c}1493335 \\
(43.4 \%)\end{array}$ & $\begin{array}{c}1613051 \\
(43.6 \%)\end{array}$ & $\begin{array}{c}1783114 \\
(45.4 \%)\end{array}$ & $\begin{array}{c}1879298 \\
(45.4 \%)\end{array}$ & $\begin{array}{c}1927921 \\
(45.1 \%)\end{array}$ & $\begin{array}{c}1929204 \\
(44.1 \%)\end{array}$ & $\begin{array}{c}1868183 \\
(43.2 \%)\end{array}$ \\
\hline Intermittent & 51922 & $\begin{array}{c}1330277 \\
(46.2 \%)\end{array}$ & $\begin{array}{c}1518176 \\
(46.8 \%)\end{array}$ & $\begin{array}{c}1592064 \\
(46.3 \%)\end{array}$ & $\begin{array}{c}1704929 \\
(46.1 \%)\end{array}$ & $\begin{array}{c}1770729 \\
(45.1 \%)\end{array}$ & $\begin{array}{c}1855608 \\
(44.8 \%)\end{array}$ & $\begin{array}{c}1946506 \\
(45.6 \%)\end{array}$ & $\begin{array}{c}1998799 \\
(45.7 \%)\end{array}$ & $\begin{array}{c}2042076 \\
(47.2 \%)\end{array}$ \\
\hline Short term & 47761 & $271405(9.4 \%)$ & $289552(8.9 \%)$ & $313591(9.1 \%)$ & $330177(8.9 \%)$ & $314794(8.0 \%)$ & $333402(8.1 \%)$ & $337862(7.9 \%)$ & $350289(8.0 \%)$ & $350149(8.1 \%)$ \\
\hline Delayed & 1683 & 40959 (1.4\%) & $54578(1.7 \%)$ & $42693(1.2 \%)$ & $52321(1.4 \%)$ & $59947(1.5 \%)$ & $70471(1.7 \%)$ & $58447(1.4 \%)$ & $92548(2.1 \%)$ & $65502(1.5 \%)$ \\
\hline None & 26613 & $0(0.0 \%)$ & $0(0.0 \%)$ & $0(0.0 \%)$ & $0(0.0 \%)$ & $0(0.0 \%)$ & $0(0.0 \%)$ & $0(0.0 \%)$ & $0(0.0 \%)$ & $0(0.0 \%)$ \\
\hline Total & 141762 & 2882241 & 3242196 & 3441682 & 3700477 & 3928584 & 4138779 & 4270736 & 4370839 & 4325910 \\
\hline
\end{tabular}




\section{WHAT IS ALREADY KNOWN ON THIS TOPIC}

The United Kingdom, along with other Western countries, has seen a substantial increase in antidepressant prescribing over the past 20 years

The reasons behind this rise are not well understood but do not seem to include lower thresholds for diagnosis or treatment, or changes in illness behaviour

\section{WHAT THIS STUDY ADDS}

The incidence of new cases of depression between 1993 and 2005 rose in young women but fell slightly in other groups such that overall incidence declined slightly

Long term prescribing accounts for the majority of antidepressant prescriptions

The rise in antidepressant prescribing seems to be largely explained by a small increase in long term prescribing
TK have received research funding from Lilly, Lundbeck, Servier, and Wyeth Pharmaceuticals. The remaining authors declare no competing interests.

1 Prescription Pricing Authority. Prescribing review-drugs used in mental health.

2007. http://www.nhsbsa.nhs.uk/PrescriptionServices/ Documents/PPDPCTReports/pctreport_20073.pdf.

2 Reid I, Cameron I. Depression: current approaches to management in primary care. Prescriber 2009;20:18-40.

3 Middleton N, Gunnell D, Whitley E, Dorling D, Frankel S. Secular trends in antidepressant prescribing in the UK, 1975-1998. J Public Health 2001;23:262-7.

4 McManus P, Mant A, Mitchell PB, Montgomery WS, Marley J, Auland ME. Recent trends in the use of antidepressant drugs in Australia, 1990-1998. Med J Aust 2000;173:458-61.

5 Helgason T, Tomasson H, Zoega T. Antidepressants and public health in Iceland: time series analysis of national data. Br J Psychiatry 2004;184:157-62

6 Hemels ME, Koren G, Einarson TR. Increased use of antidepressants in Canada: 1981-2000. Ann Pharmacother 2002;36:1375-9.

require support from their family doctor to stop medication. ${ }^{22}$ Patients not reviewed and supported are unlikely to initiate discontinuation on their own.

Long term prescribing is indicated for patients with recurrent or relapsing depression. ${ }^{23}$ Long term prescribing may, therefore, be appropriate and in line with current guidelines. ${ }^{15}$ The observed changes in antidepressant prescribing may represent better adherence to such guidelines. However, in one recent study involving detailed case review of patients on longer term antidepressant prescriptions, more than half of those examined $(56 \%)$ failed to meet criteria for a formal psychiatric diagnosis. ${ }^{24}$ Independent case review showed that there was no indication for continued receipt of an antidepressant in nearly a third $(31 \%)$ of participants. ${ }^{24}$ In another study of long term antidepressant prescribing, there was no documented mental health review over a two year period in $21 \%$ of the case notes. ${ }^{11}$

\section{Conclusions}

Antidepressant prescribing is much higher compared with 10 years ago. This increase is not because of an increase in the incidence of new cases of depression, a lower threshold for treatment, an increase in the proportion of new cases of depression for whom antidepressants are prescribed, or an increase in the duration of the prescriptions written for new cases of depression. Rather, the dramatic changes in antidepressant prescribing volumes between 1993 and 2005 seem to be largely because more patients are on long term medication and this group consumes the most drugs. In order to better understand the rise in antidepressant prescribing, research needs to focus on chronic prescribing and policy needs to focus on encouraging appropriate high quality monitoring and review of those patients who become established on long term prescriptions.

Acknowledgements: We thank Peter Smith for statistical advice and support.

Contributors: MM and TK led the data interpretation and MM the writing of the paper and is the guarantor. TK and ND conceived the original research concept and obtained the dataset from the general practitioner research database. HMY, MAM, and JM undertook the data synthesis and analysis. All contributors assisted with the journal article.

Competing interests: The data were purchased from the GPRD by the

University of Southampton, which employs all the researchers. MM and
7 In Focus: Mental Health. Pharmac [2006 Annual Review], 24.

8 Pincus HA, Tanielian TL, Marcus SC, Olfson M, Zarin DA, Thompson J, et al. Prescribing trends in psychotropic medications: primary care, psychiatry, and other medical specialties. JAMA 1998;279:526-31.

9 Olfson M, Marcus SC, Druss B, Elinson L, Tanielian T, Pincus HA. National trends in the outpatient treatment of depression. JAMA 2002;287:203-9.

10 Munoz-Arroyo R, Sutton M, Morrison J. Exploring potential explanations for the increase in antidepressant prescribing in Scotland using secondary analyses of routine data. Br J Gen Pract 2006;56:423-8.

11 Petty DR, House A, Knapp P, Raynor T, Zermansky A. Prevalence, duration and indications for prescribing of antidepressants in primary care. Age Ageing 2006;35:523-6.

12 Dunn RL, Donoghue JM, Ozminkowski RI, Stephenson D, Hylan TR. Longitudinal patterns of antidepressant prescribing in primary care in the UK: comparison with treatment guidelines. J Psychopharmacol 1999;13:136-43.

13 Hunot VM, Horne R, Leese MN, Churchill RC. A cohort study of adherence to antidepressants in primary care: the influence of antidepressant concerns and treatment preferences. Prim Care Companion J Clin Psychiatry 2007;9:91-9.

14 Garcia Rodriguez LA, Perez GS. Use of the UK General Practice Research Database for pharmacoepidemiology. Br J Clin Pharmacol 1998;45:419-25.

15 Anderson IM, Ferrier IN, Baldwin RC, Cowen PJ, Howard L, Lewis G, et al. Evidence-based guidelines for treating depressive disorders with antidepressants: a revision of the 2000 British Association for Psychopharmacology guidelines. J Psychopharmacol 2008;22:343-96.

16 Raymond CB, Morgan SG, Caetano PA. Antidepressant utilization in British Columbia from 1996 to 2004: increasing prevalence but not incidence. Psychiatr Serv 2007; 58:79-84.

17 Hyde J, Evans J, Sharp D, Croudace T, Harrison G, Lewis G, et al. Deciding who gets treatment for depression and anxiety: a study of consecutive GP attenders. Br J Gen Pract 2005; 55:846-53.

18 Hyde J, Calnan M, Prior L, Lewis G, Kessler D, Sharp D. A qualitative study exploring how GPs decide to prescribe antidepressants. $\mathrm{Br}$ J Gen Pract 2005;55:755-62.

19 Kendrick T, King F, Albertella L, Smith PW. GP treatment decisions for patients with depression: an observational study. Br J Gen Pract 2005; 55:280-6.

20 NHS Employers, BMA. Revisions to the GMS contract 2006/7. Delivering investment in general practice.

2006. http://www.nhsemployers.org/SiteCollectionDocuments/ Revisions_to_the_GMS_contract_-

21 Kendrick T, Dowrick C, McBride A, Howe A, Clarke P, Maisey S, et al. Management of patients assessed with depression severity questionnaires in UK general practice: analysis of medical record data. BMJ 2009;339:750.

22 Leydon GM, Rodgers L, Kendrick T. A qualitative study of patient views on discontinuing long-term selective serotonin reuptake inhibitors. Fam Pract 2007;24:570-5.

23 National Institute for Clinical Excellence. Management of depression in primary and secondary care. Clinical Guideline 23. 2004. http:// www.nice.org.uk/CG023.

24 Cruikshank G, MacGillivray S, Bruce D, Mather A, Matthews K, Williams B. Cross-sectional survey of patients in receipt of long-term repeat prescriptions for antidepressant drugs in primary care. Ment Health Fam Med 2008;5:105-9.

Accepted: 14 July 2009 